
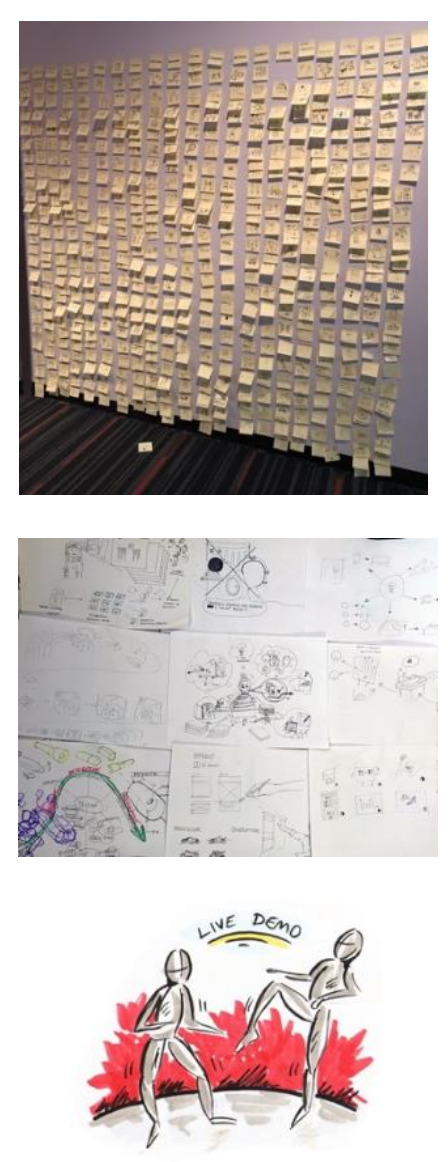

Figure 1: Visual icon dictionary CHI' 2018 [9] (top), Sketch outputs DIS' 2017 [12] (middle) and Sketching people demo by Miriam Sturdee (bottom).

\title{
So You Think You Can't Draw? A Hands-on Introductory Course on Sketching in $\mathrm{HCl}$ Techniques
}

Makayla Lewis

University of the Arts London

London, UK

Makayla.Lewis@arts.ac.uk

\section{Miriam Sturdee}

Lancaster University

Lancaster, UK

M.Sturdee@lancaster.ac.uk
Author Pre-Print - not for distribution

(C) ACM, 2020. This is the author's version of the work. It is posted here by permission of ACM for your personal use. Not for redistribution. The definitive version was published in $\mathrm{CHI} 20$ Extended Abstracts, April 25-30, 2020, Honolulu, HI, USA ACM ACM 978-1-4503-6819-3/20/04.

http://dx.doi.org/10.1145/3334480.3375046

\begin{abstract}
Hand-drawn sketching is a practice as old as our ancestors. From cave painting to picture-books, we have explored the world with our visual senses. Within Human-Computer Interaction, sketches can be used to document, ideate, and describe concepts between researcher, user, or client. Attendees will leave the course with the confidence to engage actively with sketching on an everyday basis in their research practice.
\end{abstract}

\section{Author Keywords}

Sketching, Drawing, Visual Thinking, HCI.

\section{CSS Concepts}

- Human-centered computing $\rightarrow$ Visualization $\rightarrow$ Visualization techniques.

\section{Introduction}

Sketching is a practice as old as our ancestors. From cave painting to picture-books, we have explored the world with our visual senses [3]. Now as technology develops, we are discovering ways in which the traditional visual arts can co-exist alongside the complexity of computing. Within Human Computer Interaction $(\mathrm{HCI})$, this co-existence can be embodied in 


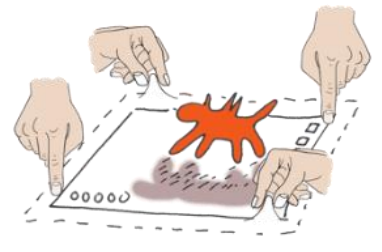

Figure 2: Hand gestures (interactions) sketch [16].

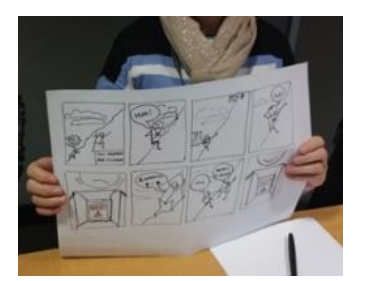

Figure 3: Participant's storyboard sketch at NordiCHI 2016.

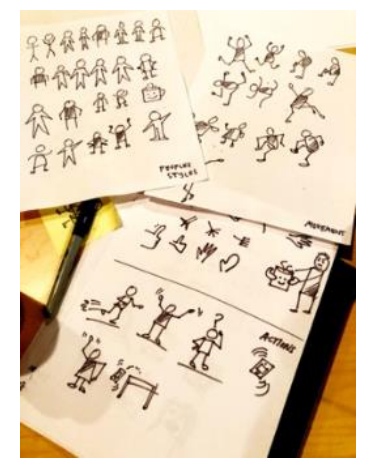

Figure 4: Sample sketching techniques (people) covered in the $C H I^{\prime} 2019$ sketching in HCI course [11]. ideation, design spaces, storytelling and impact and section of code [1], rapid prototyping [4], converted into digital representations [6], and input [7].

\section{Detailed course structure \\ Course website:}

https://sketchhci.wordpress.com/introductory-course/

Duration of course: two units, 80-minute sessions.

Course category: Methods, Hot Topic. Audience

size: 35 (maximum to 50 participants). Audience: The content of this course is suitable for individuals from industry and academia that have an interest in learning and or improving their sketching skills. Novices, experts and those with an interest are welcome to attend.

Benefits: Sketching us often overlooked in many disciplines, or referred to as a 'soft' skill, however, it can support $\mathrm{HCI}$ researchers and practitioners to ideate, collaborate, document, and explore and discover complex themes and spaces. This hands-on introductory course intends to celebrate and promote the diverse role of sketching to all practitioners, but also to generate discussion - encouraging participants to adopt sketching in their everyday research and practice. Prerequisites: No prerequisites. Course content and practical work: Participants will be guided through selected sketching techniques and strategies (Fig. 1 (middle) and 4). These techniques will be based on well-established sketching, interaction design and computer science material, e.g. [5], but will also include additional techniques and examples. Participants will also be encouraged to bring an idea which they feel would benefit from a sketching approach, in order to make a start on their own work and gain helpful feedback from the instructors and their peers. As a result of CHI 2018 [9] and CHI 2019 [11] Sketching in $\mathrm{HCI}$ course feedback, this course will consist of 7 parts and 10 hands-on activities:

1. Warm up 'The Humble Line': Activity \#1 participants will be asked to embrace their 'younger selves' by mark-making (scribbling), its purpose is to let go of perfection.

2. 'Sketchy' ice breaker: Activity \#2 participants will be given a sticky label and asked to quickly (1 minute) sketch the person next to them then give their drawing to the person. The recipient will be asked to write their name next to the sketch, then, in turn, introduce themselves (name, institution, motivation for taking part in the course, and a fun fact about themselves). The purpose is to create a safe and friendly sketching environment.

3. Sketch gallery: Exemplar presentation (e.g. Fig. 2 ) and discussion outlining visual thinking and sketching with examples from $\mathrm{HCI}$, interaction design, and computer science followed by a question and answer session to establish participants key motivations and goals of (sharing of activity \#2 outputs).

4. Visual language: Participants will sketch-along with the instructors, following a series of best 


\section{Course history}

Sketching in $\mathrm{HCI}$ courses at CHI 2018 [9] and CHI 2019 [11] and a special interest groups Sketching in $\mathrm{HCI}$ at CHI 2018 [8] and Sketching in HCI $2.0 \mathrm{CHI} 2019$ [10] has been previously given by the authors. Similar courses and workshops have been provided by the authors at: DIS 2017, NordiCHI 2016, and TReSSPASS Summer School 2016. Lewis has provided industry tuition at Design Exchange UK 2019, Agile in the City: London 2019, UX Cambridge 2018, UX in the City: Manchester 2018, UX Oxford 2017, UX Scotland 2017, UX Leeds 2017, UX Bristol 2016, UCD 2015, and TCUK 2014.

Sturdee has carried out sketching in $\mathrm{HCI}$ related research. The $\mathrm{CHI} 2020$ course will have a greater focus on developing core sketching skills and sustaining sketching in $\mathrm{HCI}$ on an everyday basis. practice examples that will be live drawn and digitally projected for immediacy: Activity \#3

5. shapes, connectors, and separators. Activity \#4 People, gestures and actions (Fig. 1 (bottom) and 4) + Show \& Tell. c) Activity \#5 Scenes including buildings, place (indoors and outdoors) + Show \& Tell. Activity \#6 icon dictionary, participants will work together to rapidly build a visual dictionary of objects and concepts present in HCI (Fig. 1 (top) and 6). Activity \#7 Typography and hand lettering, participants will explore the role of annotations and notes in sketches by hand-lettering using instructor examples (worksheets) + Show \& Tell, followed by an exemplar presentation and discussion that outlines the role of colour and shading (colour theory) in sketching.

6. Visual Storytelling: Participants will sketch individually and collaboratively (group work): Activity \#8 rapid story sketching, participants will be asked to use 'Once upon a time...' visual template, developed by the instructors, to practice quick ideation, storytelling and sketching + Show \& Tell. Activity \#9 interview your neighbour, participants will be asked to work in groups of 2 to 3 using image-interview techniques to visualize another person experience + Show \& Tell. The purpose of these activities is to bring together learnings from parts 4 and 5 .

\section{Applying Sketching in HCI: Exemplar} presentation and discussion outlining visual thinking and sketching from $\mathrm{HCI}$, interaction design and computer science (e.g. Fig. 2). Activity \#10 instructors and participants will produce a visual mind map exploring significance, benefits, and pitfalls of sketching in $\mathrm{HCI}$ and how participants could/will apply sketching into their everyday work practice.

8. 'Browse and try' resources and tools: The course will culminate in destinated area in the course room, a large table and poster board will be used to display key introductory papers, books, pens and papers, and drawing tablets. Instructors will introduce recommended resources and demonstrate tools. Participants will be given the opportunity to ask questions, browse materials, and try different sketching paper, pens, tablets, and apps.

The instructors will ensure feedback is given to each participant throughout the course. Participants will also be provided with crib sheets and practice sheets and invited to join our existing network, Sketching in $\mathrm{HCI}$ on Slack: a community engaging with sketching research and practice in $\mathrm{HCI}[8]$.

\section{Instructors biography}

Makayla Lewis is a postdoctoral research fellow at University of the Arts London, she uses participatory and visual methods to explore human factors of cybersecurity, smart money, and artificial intelligence. Makayla is also an accomplished visual thinker and sketcher who organizes the monthly SketchnoteHangout.com, SketchnoteLDN amongst other sketching events and courses, and provides visuals and sketchnotes for international companies and conferences such as CHI and ISS.

Miriam Sturdee is a Research Fellow at Lancaster University, specialising in creative practices in computer science. She holds an MFA in Visual Communication from Edinburgh college of art, and works at the 


\section{Resources}

Makayla Lewis

www.makaylalewis.co.uk contains HCI sketchnotes, daily sketches and illustrations, and links to public engagement events e.g. SketchnoteLDN, sketching crib sheets and worksheets, and an Adobe XD sketching feature. Authors coauthored ACM Interactions magazine features $[14,15]$ the latter is also ACM featured blog. Other helpful resources include Sketching User Experiences: The Workbook [7], The Sketchnote Handbook [13], and Visual Thinking: Empowering People \& Organizations through Visual Collaboration [2] books, which offer a beginner's perspective on different sketching approaches. Course notes will be produced before course and shared. Following the course, a visual summary 'sketchnotes' and crib sheet will be produced and shared. intersection of art and computing, as well investigating how sketching practice can support the development of novel technologies and public engagement.

\section{References}

[1] Ilias Bergström and Alan F. Blackwell. 2016. The practices of programming. In Visual Languages and Human-Centric Computing (VL/HCC), 2016 IEEE Symposium on, pp. 190-198.

[2] Willemien Brand. 2017. Visual Thinking: Empowering People \& Organizations Through Visual Collaboration. BIS.

[3] Neil Cohn. 2012. Explaining 'I can't draw': Parallels between the structure and development of language and drawing. Human Development 55, 4: 167-192.

[4] Matthew Cottam and Katie Wray. 2009. Sketching tangible interfaces: Creating an electronic palette for the design community. IEEE Computer Graphics and Applications 29, no. 3.

[5] Saul Greenberg, Sheelagh Carpendale, Nicolai Marquardt, and Bill Buxton. 2011. Sketching user experiences: The workbook. Elsevier.

[6] Takeo Igarashi, Satoshi Matsuoka, and Hidehiko Tanaka. 2007. Teddy: a sketching interface for 3D freeform design. In ACM SIGGRAPH Courses, p. 21. ACM.

[7] 7 - Gabe Johnson, Mark Gross, Ellen Yi-Luen Do, and Jason Hong. 2012. Sketch it, make it: sketching precise drawings for laser cutting. In CHI'12 Extended Abstracts on Human Factors in Computing Systems, 1079-1082. ACM.

[8] Makayla Lewis, Miriam Sturdee, and Nicolai Marquardt. 2018. "SketCHI: Hands-On Special Interest Group on Sketching in HCI." Extended Abstracts of the $2018 \mathrm{CHI}$ Conference on Human Factors in Computing Systems. ACM.
[9] Makayla Lewis, Miriam Sturdee, and Nicolai Marquardt. 2018. "Applied Sketching in HCI Hands-on Course of Sketching Techniques." Extended Abstracts of the 2018 CHI Conference on Human Factors in Computing Systems. ACM.

[10] Makayla Lewis, Miriam Sturdee, Jagoda Walny, Nicolai Marquardt, Thuong Hoang, Joanna Foster, and Sheelagh Carpendale. 2019. SketCHI 2.0: Hands-On Special Interest Group on Sketching in HCI. In Extended Abstracts of the $2019 \mathrm{CHI}$ Conference on Human Factors in Computing Systems (CHI EA '19).

[11] Makayla Lewis, Miriam Sturdee, and Nicolai Marquardt. 2019. Sketching in HCI: Hands-on Course of Sketching Techniques. In Extended Abstracts of the $2019 \mathrm{CHI}$ Conference on Human Factors in Computing Systems (CHI EA '19).

[12] Makayla Lewis, Miriam Sturdee, Jason Alexander, Jelle Van Dijk, Majken Kirkegård Rasmussen, and Thuong Hoang. 2017. SketchingDIS: Hand-drawn Sketching in HCI. In Proceedings of the 2017 ACM Conference Companion Publication on Designing Interactive Systems (DIS '17 Companion). ACM, New York, NY, USA, 356-359.

[13] Mike Rohde. 2013. The sketchnote handbook: the illustrated guide to visual note taking. Peachpit Press.

[14] Miriam Sturdee, Makayla Lewis, and Nicolai Marquardt. 2018. Feeling SketCHI?: the lasting appeal of the drawn image in HCI. Interactions 25, 6 (October 2018), 64-69.

[15] Miriam Sturdee, Makayla Lewis, and Nicolai Marquardt. 2018. SketchBlog \#1: the rise and rise of the sketchnote. Interactions 25, 6 (October 2018), 6-8.

[16] Sturdee, Miriam, Paul Coulton, and Jason Alexander. 2017. Using Design Fiction to Inform Shape-Changing Interface Design and Use. The Design Journal 20, no. sup1: S4146-S4157. 